\title{
Power Identification of Distributed Generation Based on Back- Propagation Neural Network
}

\author{
Fang Peng ${ }^{1 *}$, Yang Liu ${ }^{2}$, Feng Wang ${ }^{2}$, Chong $\mathrm{Li}^{3}$, Luhao Wang ${ }^{1}$, Xingong Cheng $^{1}$ and Xiju Zong ${ }^{1}$ \\ ${ }^{1}$ School of Electrical Engineering, University of Jinan, Jinan, Shandong, 250022, China \\ ${ }^{2}$ State Grid Shandong Electric Power Research Institute, Jinan, Shandong, 250002, China \\ ${ }^{3}$ State Grid Intelligence Technology Co., LTD, Jinan, Shandong, 250000, China
}

\begin{abstract}
Distributed generator (DG) is widely used and applied due to the energy and environment issues. Distributed photovoltaic generation is a typical kind of DG. Its output power is random and fluctuant, which has great influence on the safe, stable and economic operation of power system. Thus it is necessary to identify the power generated by the distributed photovoltaic generation. This paper proposes a power identification method based on BP Neural Network. The sample data comes from simulation by PSCAD and consists of current and active power that are measured in the branch of distributed network connected with DG and active power generated by the DG. The training is based on Matlab. Simulation results verify that the BP Neural Network can identify active power of DG accurately.
\end{abstract}

\section{Introduction}

Distributed generator (DG) refers to power generation facilities arranged in the distribution network or distributed near the load directly. Capacity of a small DG is usually within a few hundred $\mathrm{kW}$, while that of a large one can reach several MW [1]. Distributed generation technique is divided into photovoltaic power generation, wind power generation, combined heating, cooling and electricity (CHCP) system, fuel cell, gas turbine power generation and so on. DG can reduce transmission losses, raise energy utilization efficiency, improve voltage distribution and load power factor and strengthen reliability and economy of power grid, which makes DG become an important development direction of the power industry [2]. What is more, DG contributes to solve the problem of energy shortage and environmental pollution. The installed capacity of DG, especially photovoltaic power generation and wind power generation, increases by years due to these benefits.

As one kind of DG, distributed photovoltaic generation is widely developed and applied due to its advantage of convenient access. However, the output power of photovoltaic system is greatly affected by weather. It largely depends on the solar radiation intensity. Thus output power of the distributed photovoltaic generation is random and fluctuant, which has great influence on safe, stable and economic operation of power system. Although the DG in distribution network will not participate in voltage regulation actively, the access of it will cause magnitude and direction change of power flow, voltage deviation and voltage fluctuation. Then security of power grid is affected [3]-[7]. Knowledge of the power generated by distributed photovoltaic generation helps to grasp the change law and development trend of DG in a timely manner. It is also conducive to scientific management of DG and significant to ensure safe and stable operation of distributed network. [8] and [9] study the power flow changes of feeders with continuous distributed load after DG is connected. A three-phase power flow calculation model of the distribution network is established using the phase voltage in [10], with effect of distributed photovoltaic generation considered. [11] builds analysis model of different types of DG and calculates the power flow of distributed network using the forward-backward sweep method.

This paper uses the Back-Propagation Neural Network (BP Neural Network) to process the data of the distribution network connected with distributed photovoltaic generation, for identifying the active power generated by DG. The rest of the paper is organized as follows. Section 2 introduces structure and neuron model of the BP Neural Network. Section 3 presents the data acquisition process using PSCAD and training process using Matlab. Simulation results are also given in this section. Finally, conclusions are drawn in Section 4.

\section{Back-Propagation Neural Network}

\subsection{Structure of BP Neural Network}

BP Neural Network is a forward network with three or more layers of neurons. There is no feedback and neurons in the layers are not connected. The typical structure of BP Neural Network is shown in Figure 1.

"Corresponding author's e-mail: cse_pengf@ujn.edu.cn 
The first layer, middle layers and last layer are input layer, hidden layers and output layer, respectively. Neurons between different layers are fully connected while those included in the same layer are not connected.

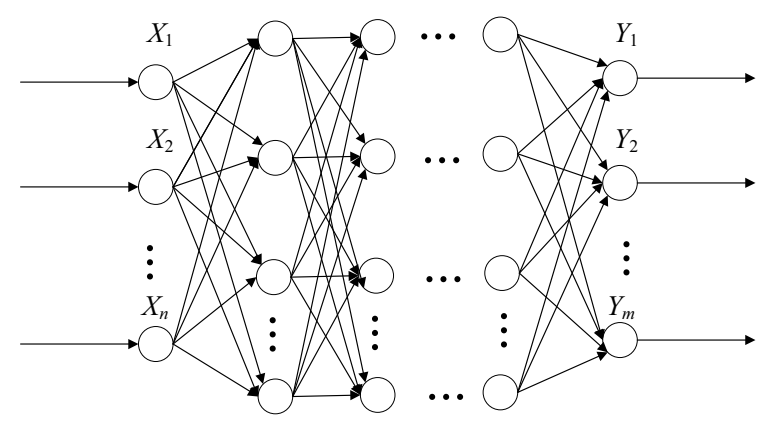

Input Layer Hidden Layer Output Layer

Figure 1. Typical structure of BP Neural Network.

BP Neural Network uses a guided method for supervision and learning. When a pair of learning patterns are provided to the BP Neural Network, activation values of the neurons propagate from the input layer to the output layer through each hidden layer. As a result, neurons in the output layer obtain the response output. By comparing the actual output and expected output of each neuron in the output layer, the error of each neuron is obtained. In the way of error reduction, connection weights between layers are corrected layer by layer from the output to the input. The process of calculating output values forward and propagating errors reverse is repeated until errors are acceptable. Then the training process ends.

\subsection{Model of calculating neurons}

For any two layers in the BP Neural Network, neurons are calculated using weight and threshold. Assume there are $n$ and $p$ neurons in the first layer L1 and second layer L2, respectively. Weight vector connecting the neurons of the two layers is assumed to be $\boldsymbol{W}=\left\{w_{i j}\right\}$, where $i=1$, $2, \ldots, n$ and $j=1,2, \ldots, p . n$ outputs of L1 turn into the input column vector $\boldsymbol{X}$ of L2, where $\boldsymbol{X}$ is $\left(x_{1}, \ldots, x_{i}, \ldots\right.$, $\left.x_{n}\right)^{\mathrm{T}}$. Thus, the input of each neuron is:

$$
s_{j}=\sum_{i=1}^{n} x_{i} w_{i j}-\theta_{j} j=1,2, \ldots, p
$$

where $\theta$ is the threshold of L2. Output $y_{\mathrm{i}}$ of L2 is determined by the transition function as (2):

$$
y_{j}=f\left(s_{j}\right)
$$

\section{Power Identification of Distributed Generation Based on BP Neural Network}

\subsection{Training process of power identification of distributed generation}

\subsubsection{Sample data acquisition.}

Sample data are obtained for training, including current and active power that are measured in the branch of distributed network connected with DG $\left(I_{\text {sum }}\right.$ and $\left.P_{\text {sum }}\right)$ and active power generated by the DG $\left(P_{\mathrm{DG}}\right)$. Active power of the branch of distributed network is decomposed to realize supervised learning. Due to the difficulty in obtaining actual data, PSCAD software is used in this part to simulate the operation state of distribution network with distributed generation. The model is shown in Figure 2. The DG is distributed photovoltaic generation.

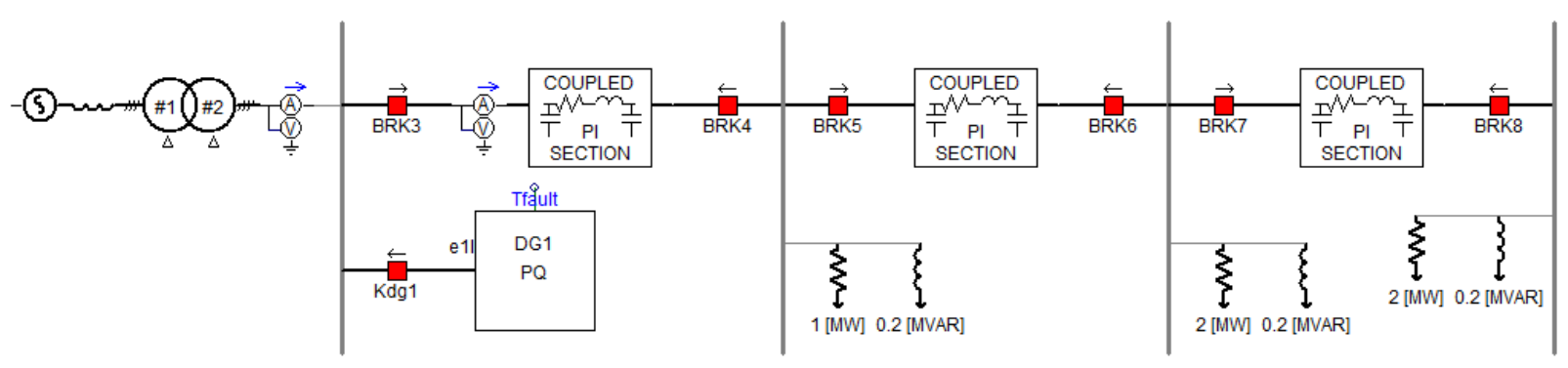

Figure 2. Simulation model of distribution network with distributed generation.

\subsubsection{Data pre-processing.}

Because distributed photovoltaic generation will cause current changes when they are connected to distribution network, Fourier algorithm is used to calculate the amplitude of the fundamental component and harmonic components of the current. The change of second harmonic component $\Delta I_{2}$ is taken as input to extract the basic characteristics of distributed photovoltaic generation. A sample data set is then established by $\Delta I_{2}$,
$P_{\text {sum }}$ and $P_{\mathrm{DG}}$, which is used for neural network training, verification and testing.

\subsubsection{Sample training.}

Matlab is used to training the sample data. A three-layer BP Neural Network is used to training sample data, as shown in Figure 3. Initialize the neural network first. Inputs and outputs of the BP Neural Network are set. Parameters of each layer can be obtained by training layer by layer from the low level to high level. Transition functions of the hidden layer and output layer are tansig() 
and purelin(), respectively. Levenberg-Marquardt algorithm is used to adjust $w_{i j}$ and $\theta_{j}$.

\subsection{Simulation results}

Based on the BP Neural Network above, identification results of active power generated by distributed photovoltaic generation are partly listed in Table 1 and the histogram of error distribution is shown in Figure 4. $P_{\mathrm{R}}$ is the active power in reality while $P_{\mathrm{I}}$ is the active power by identification. As shown in Figure 4, the identification error is less than $1 \%$ as $P_{\mathrm{R}}$ ranges from 0 MW to $2 \mathrm{MW}$. Regression coefficients of the sample set are shown in Figure 5. Regression coefficients of the training set, validation set, test set and whole sample data are equal to 1 , which verifies that the BP network can identify active power of DG accurately.

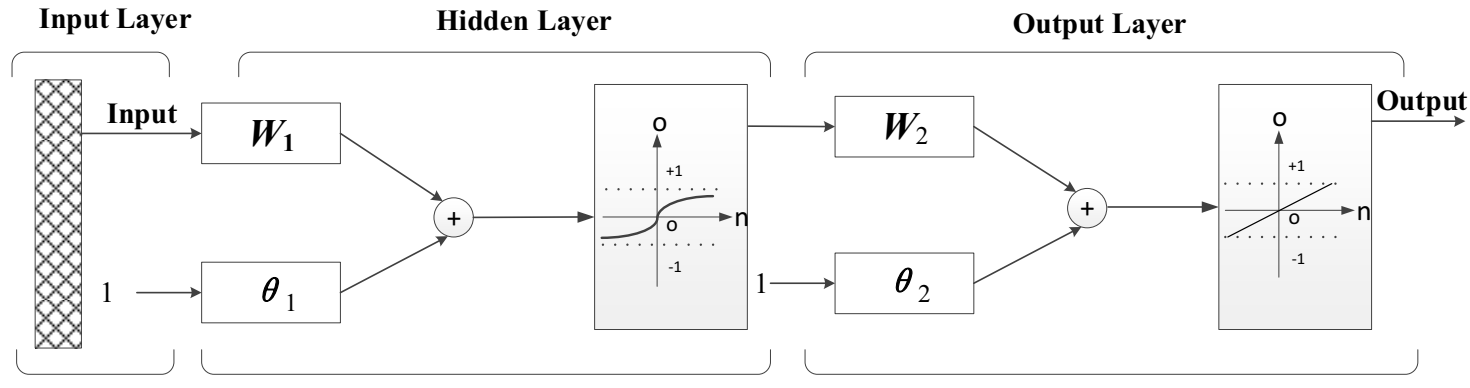

Figure 3. Simulation model of distribution network with distributed generation.

Table 1. Identification results of active power of DG based on BP Neural Network.

\begin{tabular}{l|llllllllll}
\hline $\boldsymbol{P}_{\mathrm{R}}(\mathbf{M W})$ & 0.1046 & 0.2029 & 0.3026 & 0.4021 & 0.5017 & 0.6017 & 0.7000 & 0.7994 & 0.8978 & 0.9967 \\
$\boldsymbol{P}_{\mathbf{I}(M W)}$ & 0.1039 & 0.2043 & 0.3018 & 0.3983 & 0.4977 & 0.6000 & 0.7003 & 0.8013 & 0.8990 & 0.9976 \\
\hline $\boldsymbol{P}_{\mathrm{R}}(\mathbf{M W})$ & 1.0963 & 1.1946 & 1.2935 & 1.3915 & 1.4904 & 1.5891 & 1.6884 & 1.7865 & 1.8854 & 1.9846 \\
$\boldsymbol{P}_{\mathbf{I}(\mathbf{M W})}$ & 1.0958 & 1.1936 & 1.2924 & 1.3942 & 1.4897 & 1.5917 & 1.6908 & 1.7872 & 1.8859 & 1.9832 \\
\hline
\end{tabular}



Figure 4. Simulation model of distribution network with distributed generation.
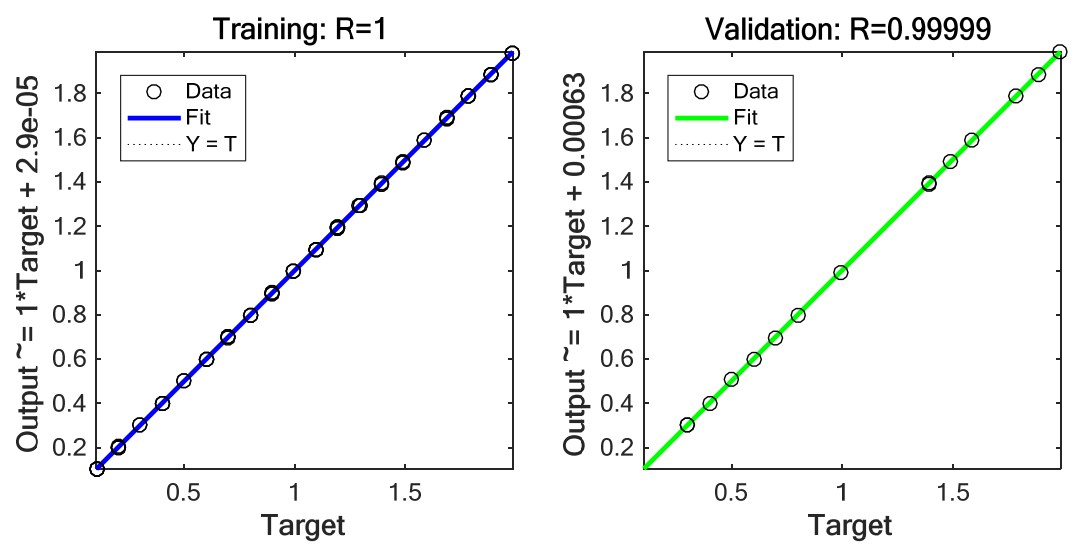

(a) Regression coefficients of the training set, validation set. 

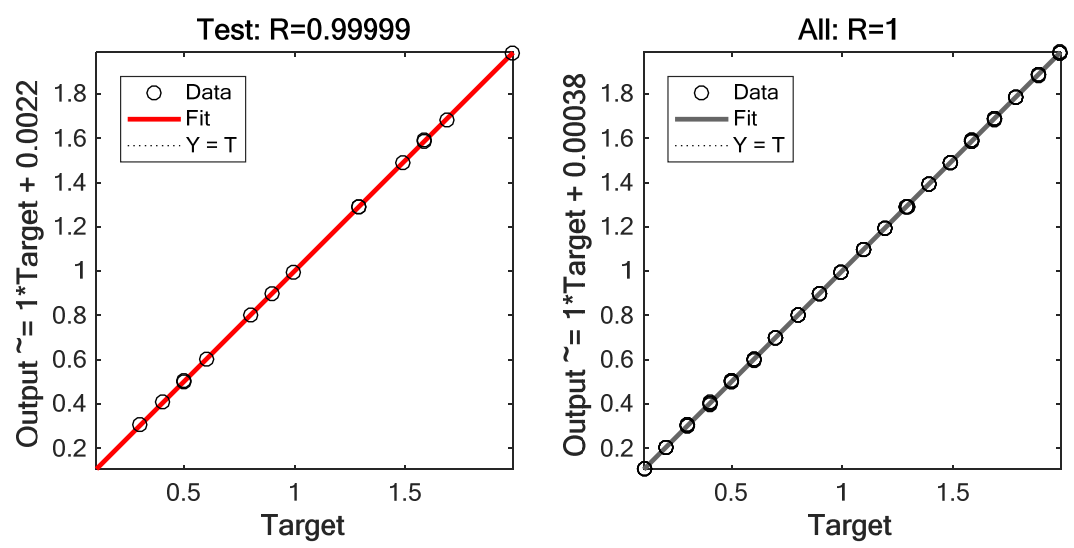

(b) Regression coefficients of the test set and whole sample data.

Figure 5. Regression coefficients of the sample set.

\section{Conclusion}

The output power of DG is important for stable operation of power system. To grasp the change and development trend of power generated by DG, this paper proposes a power identification method based on BP Neural Network. The BP Neural Network used includes three layers, which are input layer, hidden layer and output layer. The sample data comes from simulation by PSCAD and consists of current and active power that are measured in the branch of distributed network connected with DG and active power generated by the DG. The training is based on Matlab. Weight values and threshold values are adjusted during the process of error back propagation. Simulation results verify that the BP Neural Network can identify active power of DG accurately.

\section{Acknowledgments}

This work was supported in part by the Doctoral Foundation of University of Jinan of China (XBS1839), in part by the Project of State Grid Shandong Electric Power Company (52062618001S), in part by the Project of Shandong Province Higher Educational Youth Innovation Science and Technology Program (2019KJN029) and in part by the National Science Foundation of China (61803174).

\section{References}

1. Wang, C.S., Li, P. (2010) Development and challenges of distributed generation, micro-grid and smart distribution system. Automation of Electric Power Systems, 34: 10-14, 23.

2. El-khattam, W., Salama, M.M.A. (2004) Distributed generation technologies, definitions and benefits. Electric Power Systems Research, 71: 119-128.

3. Wang, J., Chen, J., Liu, J., Xu, Y., Yang, F., Tang, H. (2015) Novel immune clonal algorithm for multitypes distributed generators planning. Proceedings of the CSU-EPSA, 27: 21-28.
4. Zou, H., Han, X., Liao, Q., Liu, D., Zhu, Z., Chen, W. (2016) Penetration capacity calculation for distributed generation considering voltage quality and short circuit capacity constraints. Power System Technology, 40: 2273-2280.

5. Wang, S., Song, L., Shu, X. (2019) Adaptive overcurrent protection of active distribution network with high penetration of distributed generations and multiple loads. High Voltage Engineering, 45: 17831794.

6. Huang, W., Liu, J., Wei, H., Zhang, Z. (2015) Extreme capacity limitations of photovoltaic generators in distribution grids. Power System Protection and Control, 43: 22-28.

7. Wang, C., Zhang, N., Zhao, J., Sun, C., Ke, X., Wang, J. (2016) Maximum Capacity Calculation of Distributed Generators Considering Fluctuation Characteristics. Proceedings of the CSU-EPSA, 28: 38-43.

8. Wang, Z., Zhu S, Zhou S., Huang R., Wang L. (2005) Study on location and penetration of distributed generations. Proceedings of the CSU-EPSA, 17: 5358.

9. Wang, C., Nehrir, M.H. (2004) Analytical approaches for optimal placement of distributed generation sources in power systems. IEEE Transactions on Power Systems, 19: 2068-2076.

10. Alam, M.J.E, Muttaqi, K.M., Sutanto, D. (2013) A three-phase power flow approach for integrated 3wire MV and 4-wire multigrounded LV networks with rooftop solar PV. IEEE Transactions on Power Systems, 28: 1728-1737.

11. Chen, H., Chen, J., Duan, X. (2016) Power flow calculation of distribution network with distributed power supply. Automation of Electric Power systems, 30: 35-40. 